\title{
Perpendicular Deformation of a Near-Single-Crystal Triblock Copolymer with a Cylindrical Morphology. 1. Synchrotron SAXS
}

\author{
Christian C. Honeker, Edwin L. Thomas,* Ramon J . Albalak, Damian A. Hajduk, \\ Sol M. Gruner, and Malcolm C. Capel
}

Department of Materials Science \& Engineering, Massachusetts I nstitute of Technol ogy,

77 Massachusetts Avenue, Room 13-5094, Cambridge, Massachusetts 02139

Received April 3, 2000; Revised Manuscript Received September 25, 2000

\begin{abstract}
A poly(styrene-block-isoprene-block-styrene) (SIS) triblock copolymer with a polystyrene (PS) cylinder morphology was processed via roll-casting to produce a near single-crystal texture. Deformation experiments normal to the cylinder axis were carried out using synchrotron small-angle $X$-ray scattering (SAXS) with the beam both parallel and perpendicular to the cylinder axis. In situ measurement of load and displacement enabled morphological information to be mapped to the stressstrain curve. Results indicate that the deformation proceeds in two stages. Deformation to strains of approximately $100-130 \%$ is nearly affine. The rubber matrix extends al ong the stretching direction (SD) while contraction occurs almost exclusively along the neutral direction due to the constraint imposed by the aligned PS cylinders. A measured Poisson's ratio of 0.9 compares favorably with a value of 1 expected for a perfect composite. At deformations beyond $130 \%$ an X-pattern is observed at perpendicular incidence. The angle between the arms of the $X$ increases asymptotically, while the intercylinder spacing remains fairly constant. The initial hexagonal pattern undergoes a continuous distortion and provides further evidence for the deformation discontinuity in the rate of lateral contraction. The morphological observations in reciprocal space are interpreted in terms of a kinking instability in which the plane strain state imposed by the oriented cylinders is relieved by their kinking into a chevron pattern. The kinking transition strain is expected to be dependent on the degree of cylinder misorientation, the shear resistance of the rubber matrix, and the bending resistance of the PS cylinders.
\end{abstract}

\section{Introduction}

1.1. Motivation. Styrenic-based thermoplastic elastomers (TPEs) make up approximately $50 \%$ of the multibillion dollar TPE industry. ${ }^{1,2}$ This market is expected to grow rapidly (8\%/year) as TPEs replace conventional vulcanates. The use of conventional thermoplastic processing techniques including extrusion, injection-molding, and blow-molding is one major advantage in the application of TPEs. ${ }^{3}$ The strong flowfields inherent in these techniques often introduce orientation in TPE morphology which has ramifications for a variety of properties in the final product. ${ }^{4-6}$ Elucidation of the relationship between the oriented morphology and the anisotropic mechanical properties of processed TPEs is thus necessary for practical reasons.

Styrenic TPEs are typically based on linear styrenediene-styrene triblock copolymers with styrene contents ranging from $10 \%$ to $90 \%$ depending on application. As the composition is varied, morphologies analogous to the familiar diblock copolymer morphologies ${ }^{7-11}$ are obtained. With increasing styrene content, these are styrene spheres in a bcc arrangement, followed by hexagonally packed styrene cylinders, a tricontinuous structure, and lamellae. The morphologies invert at high (>50\%) styrene content. The degree of mechanical anisotropy depends on the symmetry and connectedness of the styrene domains as well as the degree of microstructural order. With increasing styrene content, the minority domains gain in dimensionality-spheres as $0-D$, cylinders as 1-D, lamellae as 2-D, and bicontinuous as 3-D reinforcing structures. Recent studies have begun to clarify the impact of morphological anisotropy in macroscopically oriented specimens with spherical, ${ }^{12}$ double gyroid, ${ }^{13}$ and lamellar ${ }^{14}$ morphologies on mechanical properties. Here, we describe such an examination for the cylinder morphology deformed perpendicular to the cylinder axis to large deformations.

1.2. Previous Work on Oriented Cylinder Morphologies. As the most common styrenic TPEs, triblock copolymers with the polystyrene (PS) cylinder morphology (20-30 wt \% PS) have been the most studied morphology in terms of mechanical properties. However, there are far fewer investigations on oriented cylinders. Keller and co-workers, in a series of ground breaking studies on extruded cylinders, made the important connection that mechanically, highly oriented block copolymer TPEs can be treated as nearly perfect composite materials. ${ }^{15} \mathrm{~A}$ commercial poly(styrene-butadiene-styrene) (SBS) (Kraton 102 Shell) with a total molecular weight of $85 \times 10^{3}$ and a PS weight fraction of 0.25 was extruded at temperatures above the glass transition of the PS phase $\left(\mathrm{T}_{\mathrm{g}}^{\mathrm{PS}}\right)$ through an orifice followed by annealing. ${ }^{16}$ The macroscopically oriented samples were examined in a variety of experiments which have been reviewed. ${ }^{15}$ The small-strain deformation behavior was described in terms of the elastic compliance tensor for a hexagonally symmetric material. The dependence of the sample stiffness as a function of angle between the stretching direction (SD) and the cylinder axis was fit using several fiber reinforcement models. ${ }^{17}$ The structural response to applied deformation was examined using a variety of techniques mainly for deformation parallel to the cylinder axis (strains < $120 \%)$. SAXS experiments investigated the deformation along a direction perpendicular to the cylinders (strain $<50 \%)^{18}$

One of the first uses of a reciprocating shear apparatus, which consists of two parallel plates moving 


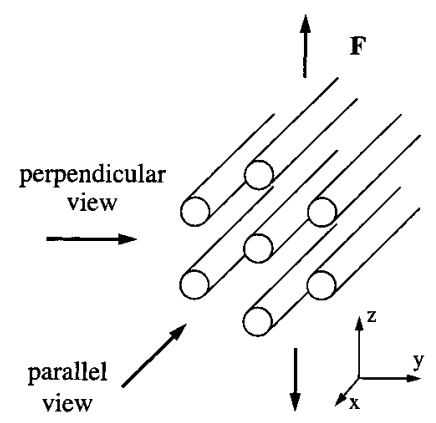

Figure 1. Perpendicular deformation geometry. The sample is stretched in the $z$ direction (vertical) with the X-ray beam incident in either the $x$ direction parallel to the cylinder axis (parallel view) or in the $y$ direction perpendicular to the cylinder axis (perpendicular view).

slowly with respect to one another, was to prepare highly oriented SIS triblock copolymers for deformation investigations. ${ }^{19}$ The samples had a near-single-crystal texture as determined by 2-D SAXS.20 Perpendicular deformation experiments were performed using 2-D SAXS with the incident beam al ong the cylinder axis. ${ }^{21}$ Measurement of the sample and lattice dimensions in real and reciprocal space, respectively, indicated an affine deformation up to the highest strain investigated (50\%). U pon unloading, the sample completely recovered to its initial state.

The first large strain (>200\%) study of the oriented cylinder morphology revealed several important observations. ${ }^{22} 2-\mathrm{D}$ SAXS with the incident beam normal to the cylinders (Figure 1) resulted in a four-point pattern at deformation $\geq 130 \%{ }^{23}$ This distinctive pattern was interpreted as a zigzag arrangement of bent cylinders, the degree of bending increasing with deformation. Viewing along the cylinder axis yielded additional information about the 3-D nature of the deformation. The hexagonal pattern distorts, so that all reflections along the SD and perpendicular to the SD move away from and toward the origin, respectively. A schematic representation of the 3-D diffracted intensity distribution at $130 \%$ deformation was attempted, but neither a 3-D real space model to accompany this 3-D diffraction data nor a 3-D representation of the available diffraction data at higher deformations (> 130\%) was provided.

A second SAXS study on the oriented PS cylinder morphology concluded that, at the highest deformations, orientation of the bridging molecules controls deformation. ${ }^{24}$ The PS domains assume essentially the same chevronlike configuration of bent cylinders irrespective of the deformation direction. This universal high deformation state was postulated for deformation directions parallel, perpendicular and at $45^{\circ}$ to the cylinder axis on the basis of schematic 2-D SAXS patterns constructed from 1-D SAXS data. The structural models for the deformed states were 2-dimensional, providing no information concerning possible deformation processes out of the detection plane.

The morphological response to small oscillatory strain $(<6.7 \%)$ via synchrotron SAXS ${ }^{25}$ of a press-molded poly(styrene-ethylene-butylene-styrene) triblock copolymer found that the cylinder spacing responded in a nonaffine manner for both deformation parallel and perpendicular to the cylinder axis. This nonaffine response was attributed to contributions to the strain by misoriented grains which rotate into the stretching direction during deformation. It should be noted that both the type of deformation (oscillatory strain) and the microstructure of the rubbery midblock (hydrogenated butadiene) differed from most previous deformation studies of oriented triblocks.

A recent analytical and finite element mechanics (FEM) treatment of the buckling phenomenon in two dimensions under plane strain conditions (relevant to for both lamellar and cylindrical TPEs) has provided insight into the nature of the undulation instability. ${ }^{26}$ The microphase texture (the multilayered structure) is coupled to the macroscopic strain fields via two mesoscale moduli, associated with hard phase bending and displacement of the soft phase relative to the hard phase. Perpendicular deformation of perfectly aligned multilayered structures causes an initial sinusoidal buckling, which rapidly develops into a chevron texture at higher strains. In this model the structure is stabilized against the buckling instability by the shear modulus of the matrix. The buckling instability is predicted to correspond to a sharp turnover in the stress-strain curve. It was found that the onset of the buckling instability as well as the buckling wavelength is very sensitive to the boundary conditions. Defects associated with real materials, such as layer misorientation, grain boundaries, or dislocations, are expected to play a role in determining the onset and dimensions of the buckling instability.

1.3. Organization of the Paper. In the present work, the evolution of the microdomain morphology during deformation is followed by in situ 2-D synchrotron SAXS. The incident beam direction was varied in order to probe the morphology in two principal directions: parallel and perpendicular to the cylinder axis (Figure 1). The initial degree of orientation has important implications for the deformation behavior and is considered first. Representative deformation experiments in the perpendicular and parallel viewing directions follow. The data are divided into three deformation regimes: small strain, transition, and large strain. Emphasis is placed on the large strain results. A discussion of the deformation mechanism in terms of kinking concludes the paper. A detailed structural model for the evolution of the deformed morphology consistent with the SAXS data presented here is provided by extensive transmission electron microscopy (TEM) in the accompanying paper. ${ }^{27}$

\section{Experimental Section}

The poly(styrene-isoprene-styrene) (SIS) triblock copolymer was obtained from Dexco (Dexco Polymers, 12012 Wickchester, Houston, TX 77079). The triblock has a composition of 29 wt \% PS ( $26 \mathrm{vol} \%$ ) as determined by NMR and a total molecular weight of $101 \times 10^{3}$ as determined by GPC. Together this information yields block molecular weights of (15-7215) $\times 10^{3}$. The isoprene midblock has a microstructure consisting predominantly of 1,4 addition (92\%). SAXS analysis of the cylindrical morphology provides an intercylinder spacing of $310 \AA$, which together with the known volume fraction yields a cylinder diameter of $83 \AA$.

2.1. Roll-Casting and Sample Preparation. Oriented films were prepared by the roll-casting technique, ${ }^{28}$ the essential features of which are shown in Figure 2. Two parallel rollers, one of stainless steel and the other of Teflon, are driven by two independent motors and separated by a micrometercontrolled gap. The procedure used to prepare oriented films is as follows. The gap and roller speeds are set to typical values of $0.05-0.4 \mathrm{~mm}$ and $9 \mathrm{rpm}$ (shear rate of $0.14 \mathrm{~s}^{-1}$ ) respectively. Films of thicker dimensions are prepared by increasing the gap and adding more solution step-wise. A concentrated solution (1 weight of polymer/2 volumes of solvent) is intro- 


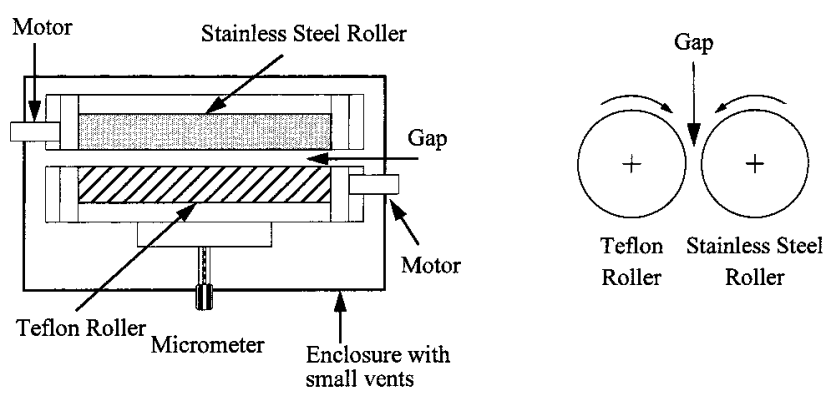

Figure 2. Schematic of the roll-caster used to generate macroscopically oriented films. Two independent, motorcontrolled, parallel rollers counterrotate at a constant frequency. The rollers are separated by a micrometer-controlled gap. A viscous block copolymer solution is introduced into the gap region and allowed to undergo microphase separation in the presence of the flow field. After slow evaporation of the solvent an oriented film preferentially forms on the stainlesssteel roller.

duced into the gap region so that both rollers are uniformly coated. A box is placed over the roll caster to slow solvent evaporation. The finished film is allowed to dry before removal and annealing.

In addition to the gap setting and roller speed, important processing variables include the concentration of the solution and evaporation rate. Here a concentration of approximately $33 \%$ of polymer in cumene is used. The concentration is chosen as a compromise between the speed of dissolution and solution viscosity. Higher polymer concentrations take longer to prepare and are closer to the solution order-disorder transition (ODT). Lower polymer concentrations require smaller gap settings to prevent escape of solution from the gap. Cumene, like toluene, has been found to be a nonpreferential solvent for the poly(styrene-isoprene) (SI) block copolymer system ${ }^{29}$ with the additional property of having a lower vapor pressure. ${ }^{30}$ Sl ower solvent evaporation leads to an extended period of orienting during film formation at the expense of a somewhat prolonged drying time.

In addition to residual solvent, freshly roll-cast films contain a significant amount of residual strain on account of a net preferential chain orientation along the flow direction. ${ }^{31}$ Drying and annealing rids the film of both the residual solvent and strain as well as increases the degree of orientation. The films are held between Teflon sheets separated by a spacer to facilitate unconstrained dimensional relaxation. Films are dried under vacuum for 1-2 days before the temperature is ramped to $120^{\circ} \mathrm{C}$ for $5-7$ days.

2.2. Synchrotron SAXS. Small-angle X-ray scattering (SAXS) was performed on beamline $X-12 B$ at the National Synchrotron Light Source (NSLS) in the Brookhaven National Laboratory (BNL). The X-ray wavelength was $1.549 \AA$ while the sample to detector distance (SDD) varied from 150 to 250 $\mathrm{cm}$. Conversion of detector pixels to the scattering vector $\mathbf{q}$ $\left(\AA^{-1}\right)$ was performed with the help of cholesteryl myristate ${ }^{32}$ as primary calibrant and a dried and stained duck tendon as secondary calibrant. Data obtained from the 2-D positionsensitive gas-proportional detector (PSD) was corrected for nonuniformity in response using procedures described at http:// crim12b.nsls.bnl.gov/x12b_det.html. Since scattering was strong in comparison to the background and only peak positions (not intensity) were extracted from the data, background was not subtracted from the raw 2-D SAXS data. Indexing of the directions and planes in this hexagonal system will be performed using the hkil notation in order to emphasize symmetry. ${ }^{33}$ The HKL notation for hexagonal symmetry is equivalent and necessary to perform crystallographic calculations. ${ }^{34}$

2.3. Deformation Stage. Deformation experiments were carried out using a custom built deformation stage while simultaneously acquiring SAXS data. The deformation stage applies symmetrical strain via a single stepper motor and measures load via a $5 \mathrm{lb}$ load cell. Maximum travel is limited to approximately $90 \mathrm{~mm}$. Load was recorded on a strip-chart

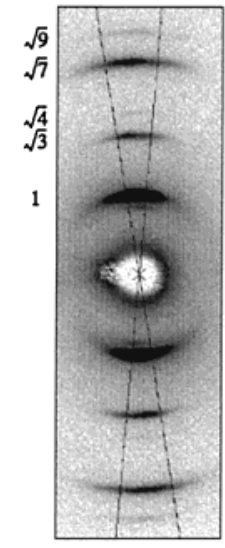

(a)

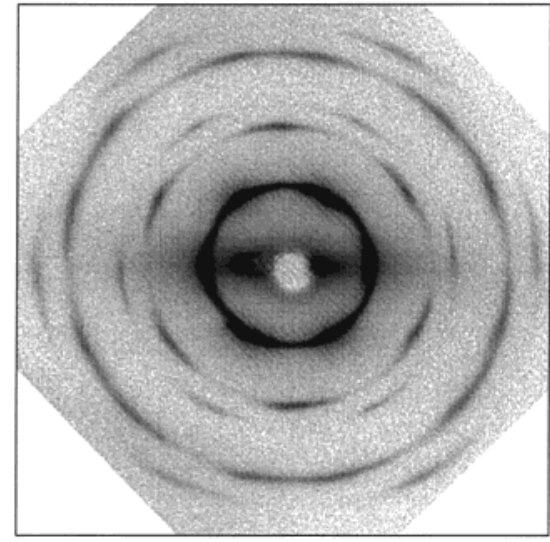

(b)
Figure 3. 2-D SAXS patterns of the perpendicular (a) and parallel (b) views demonstrating the initial degree of orientation in an unstretched sample. The crossed lines through the origin in a indicate the range of azimuthal integration used for Figure 9a.

recorder and subsequently digitized and divided by initial cross-sectional area to yield engineering stress. Engineering strain was determined from the number of steps from the stepper motor divided by the initial gage length. Samples were stamped from roll-cast films of 1-2 mm thickness using steelruled dies of varying widths taking care to properly align the flow direction perpendicular to the specimen long axis. Typical deformation parameters include strain rates of $0.5-1 \mathrm{~s}^{-1}$, gauge lengths of $5-15 \mathrm{~mm}$, and cross sections of $1-2 \times 1-3$ $\mathrm{mm}^{2}$.

\section{Initial Orientation}

3.1. Perpendicular View. SAXS patterns of the sample in the unstretched state (Figure 3) clearly show a high degree of morphological orientation induced by the roll-casting process. Figure 3a shows a 2-D SAXS pattern of an oriented SIS sample in the perpendicular viewing geometry. The series of peak maxima (labeled in sequential order by their respective peak position ratios $q_{n} / q_{1}$ ) indicates that the cylinders are highly aligned in the flow $(x)$ direction. It is apparent that the peaks have differing intensities as a consequence of form factor modulation (see bel ow).

The degree of cylinder misorientation about the y axis can be quantified by the azimuthal width of the peak intensity. Perfect cylinder orientation along $x$ corre sponds to no azimuthal width (delta functions), while perfect misorientation results in an azimuthal width of $360^{\circ}$ (rings). The azimuthal full-width at half-maximum (fwhm) of the $\sqrt{3}$ peak in Figure $3 a$ is approximately $15^{\circ}$.

A second statement of the degree of orientation can be made on the basis of Figure 3a. As demonstrated in Figure 4, the scattering pattern in Figure 3a should not exhibit all possible peak ratios for the hexagonal cylinder lattice if the morphology were perfectly oriented. Since all allowed reflections for a sample with p6mm symmetry are observed, one can conclude that the 2-D packing of the cylinders is not that of a perfect single crystal. The axial misorientation or misorientation of the cylinder lattice about the $x$ axis accounts for the observation of all possible peaks for the cylinder morphology in Figure 3a. The intensities of the peaks in Figure 3a are thus determined not only by the form factor (see below) but also by the extent of rotational 

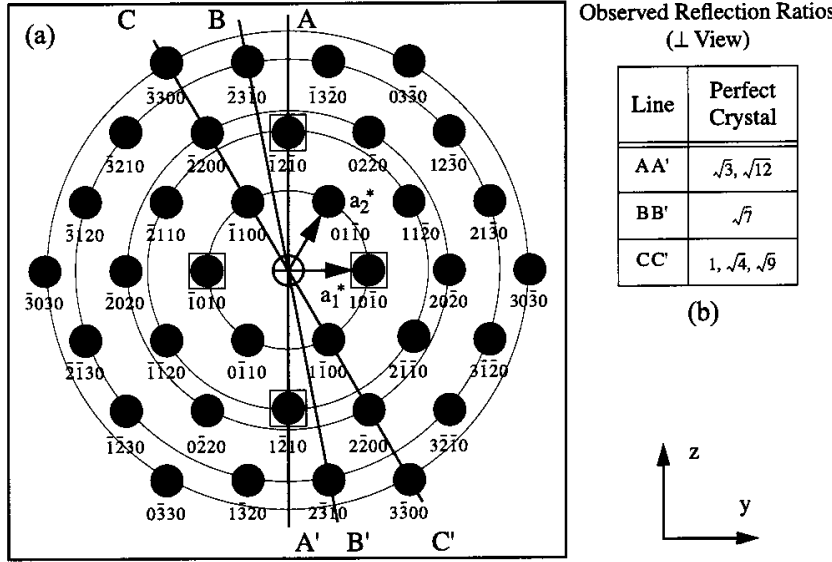

(b)

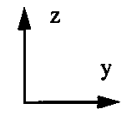

Figure 4. (a) Indexed (hkil) section of reciprocal space of a lattice with symmetry p6mm. The black dots refer to the diffracted peak positions of a perfect crystal, while the rings refer to the intensity distribution of a uniaxial (fiber-symmetric) sample. With increasing radii the rings represent spacing ratios of $1, \sqrt{3}, \sqrt{4}, \sqrt{7}$, and $\sqrt{9}$. The $(1 \overline{2} 10)$ and (12) 10$)$ pair which belong to the $\sqrt{3}$ family and the (1010) and (1010) pair which belong to the $\sqrt{1}$ family are aligned along and normal to the stretching direction, respectively. The peaks tracked in subsequent analysis are boxed. Note that the film surface is parallel to the closest-packed $\{10 \overline{1} 0\}$ planes and that the stretching direction (SD) is vertical. (b) Table listing the expected reflections for indicated traces of the E wald sphere (lines $A A^{\prime}, B B^{\prime}, C C^{\prime}$ ) passing through the origin of the diagram at left. Line $A^{\prime} A^{\prime}$ corresponds to the perpendicular view geometry. Note that it is possible for a sample with a cylinder morphology to exhibit a reflection for every allowed $\left|q_{\text {hkil }}\right|$ when X-rays are incident normal to the cylinders, even if the azimuthal misorientation is not complete (see Figure $3 \mathrm{~b}$ ).

misorientation. SAXS patterns in the perpendicular view geometry of samples oriented via the roll-caster are a composite of responses from grains of cylinders with a distribution of orientations about their axes. The extent of this rotational misorientation can best be determined by SAXS in the parallel view geometry.

3.2. Parallel View. Figure $3 b$ demonstrates that the morphological order far exceeds that of a uniaxial fiber (represented by the rings of Figure 4) and approaches that of a single crystal (represented by the dots in Figure 4). Here the SAXS pattern obtained in the parallel view geometry yields direct information on the degree of rotational misorientation. Most grains of cylinders are predominantly aligned in such a way that the closestpacked planes $((10 \overline{10}))$ are parallel to the film edge. Measurement of the azimuthal width of the peaks in Figure $3 b$ yield fwhm values between 10 and $13^{\circ}$.

The diffraction pattern in Figure $3 b$ is made up of a hexagonal lattice modulated by the form factor of a cylinder. The interrelationship of the lattice factor with the form factor can best be demonstrated for an unoriented sample in Figure 9b. Here, the relative intensities of the peaks associated with the lattice are shown modulated by the envelope of the form factor for a cylinder of radius $R=83 \AA$. The peak positions are simulated for a hexagonal lattice with a lattice constant of $310 \AA$ appropriate for a volume fraction of 0.26 . It is clear that the first zero of the form factor (at $q=0.046$ $\AA^{-1}$ ) occurs between the $\sqrt{3}$ and $\sqrt{4}$ peaks while the second zero effectively suppresses the $\sqrt{12}$ peak.

\section{Results}

4.1. Perpendicular View Deformation Series. Figure 5 shows a stress-strain plot of a roll-cast SIS

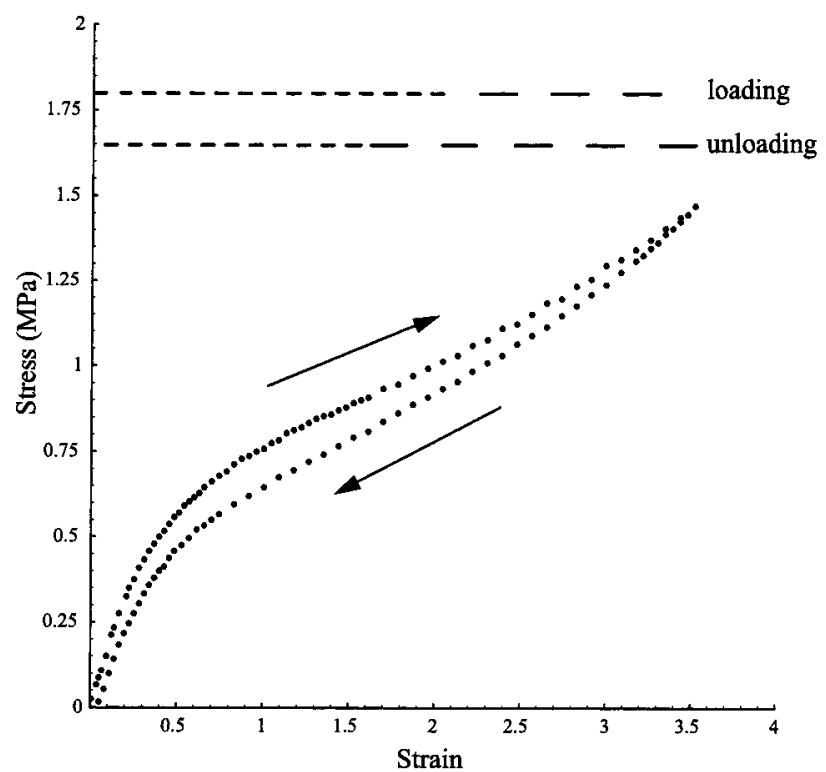

Figure 5. Stress-strain plot corresponding to the SAXS data of F igure 6 for a sample stretched perpendicular to the cylinder axis (Figure 1 ) at a strain rate of $0.5 \mathrm{~s}^{-1}$. The dashed lines at the top indicate data acquisition periods for the loading and unloading cycles, respectively. Larger exposure times are required for patterns taken at high deformation because of sample thinning due to the Poisson effect. The short and long dashes correspond to 10 and 30 s exposures, respectively.

sample with a cross-sectional area of $3.3 \mathrm{~mm}$ wide $x$ $1.6 \mathrm{~mm}$ thick. The horizontal dashed lines at the top indicate the SAXS sampling frequency on loading and unloading. The morphology undergoes a continuous change during the constant strain-rate deformation, so that each recorded SAXS pattern represents an integration over a finite time (equivalent strain increment). This means that each recorded SAXS pattern is made up of an evolving diffraction pattern. All SAXS measurements as a function of deformation will therefore be taken with respect to the average strain during the course of a given SAXS pattern acquisition.

SAXS patterns corresponding to the loading portion of the stress-strain plot in Figure 5 are shown in Figure 6. For each deformation two images of different intensity threshol ds are displayed. Qualitatively, one can discern two deformation regimes. At relatively low strains $(<100 \%)$ the patterns remain essentially 1-D with the peaks aligned along the meridian. With increasing deformation these peaks broaden and the intensity distribution splits into a characteristic " $X$ ". The X-angle is defined by the angle between pairs of reflections normal to the cylinders (see Figure 14b). Note that the structure giving rise to the pattern at $320 \%$ strain is highly ordered as there are several $(>4)$ orders present in each arm. An explanation of the morphological changes giving rise to these diffraction signatures is provided in the discussion.

4.2. Parallel View Deformation Series. Figure 7 shows a strain-strain plot of a roll-cast SIS sample with a cross section $1.2 \mathrm{~mm}$ wide $\times 1.9 \mathrm{~mm}$ thick. The reduced cross section of this particular sample generates a higher stress at a given strain than for the sample in Figure 5. The sample has also been stretched to a higher deformation due to the choice of a smaller initial gauge length. The same sampling frequency as that used for the experiment in Figure 5 is employed, resulting in a larger strain increment during a given exposure time. 


\section{Perpendicuar View}

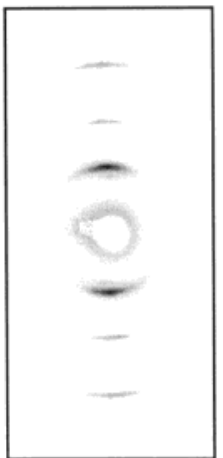

(a)

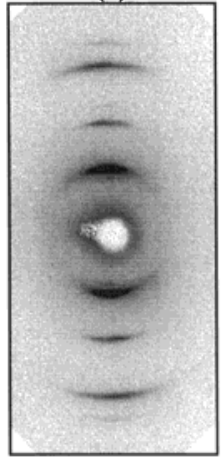

$0 \%$

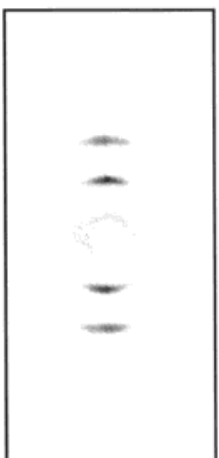

(b)

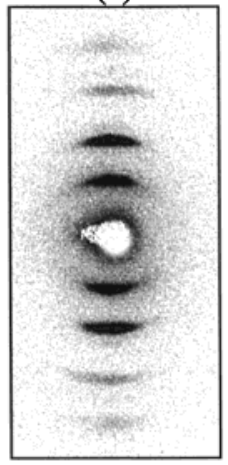

$20 \%$

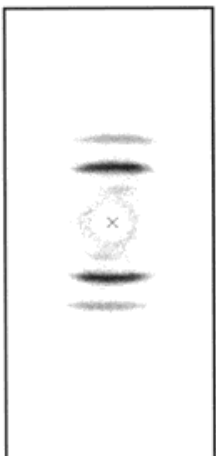

(c)

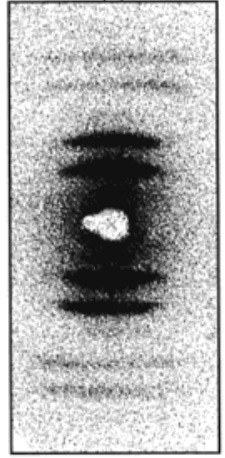

$100 \%$

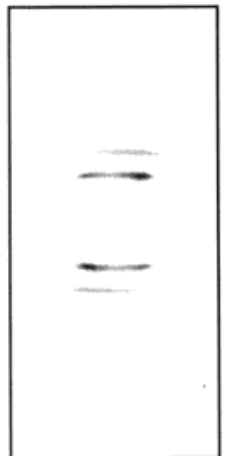

(d)

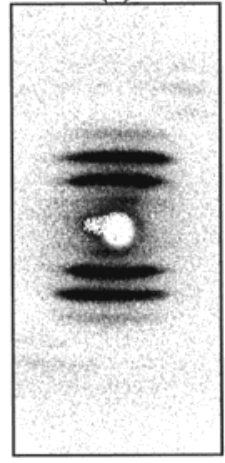

$145 \%$

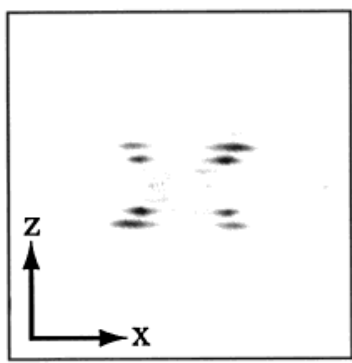

(e)
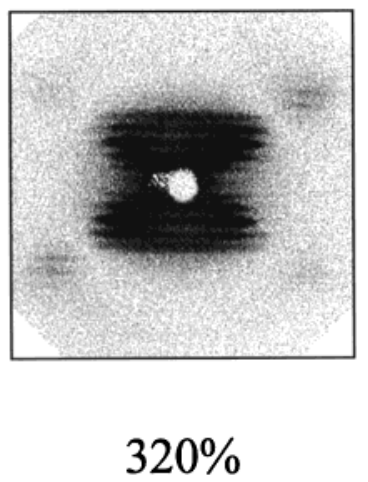

Figure 6. 2-D SAXS as a function of strain for an SIS triblock cylinder morphology undergoing perpendicular deformation in the perpendicular view geometry (see Figure 1). With increasing deformation the peaks making up the 1-D intensity profile move toward the origin, change in relative intensity, and broaden until at approximately $130 \%$ strain they split in two. Thereafter, a characteristic " $X$ " pattern devel ops (here shown at 320\% strain). Even at the highest deformations there are several peaks making up each arm. The structure at these high deformations remains highly ordered.

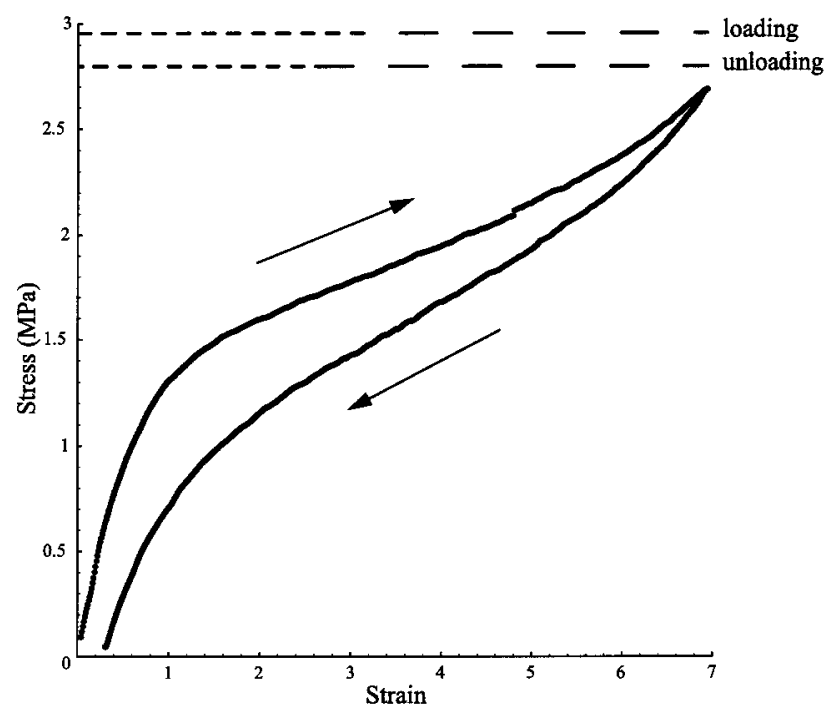

Figure 7. Stress-strain plot corresponding to the SAXS data of Figure 8. The dashed lines at the top indicate data acquisition periods for the loading and unloading cycles, respectively. A smaller cross-section and shorter gauge length account for the higher stress and strain compared to Figure 5.

As the cylinders are deformed normal to their axes, the hexagonal pattern shown in Figure 8a changes continuously. Those peaks along the SD move toward the origin, while the peaks normal to SD move away from it. The remaining peaks move according to the magnitude of their components along and normal to the SD, respectively. The rings of diffraction seen in Figure $8 \mathrm{a}$ are immediately distorted into ellipses (Figure 8b). The hexagonal symmetry of the cylinder packing is broken by the deformation. The symmetry of the re maining patterns about the origin during the course of the deformation is that of point group $2 \mathrm{~mm}$.

As in the perpendicular view geometry experiment, the intensities of the observed peaks are modulated by the form factor. In Figure 8, parts $b$ and $c$, the $\sqrt{3}$ peaks along the SD, the (1210) and (1210) peaks, according to the indexing of Figure 4, gain in intensity as they move away from the zero of the form factor (indicated by the circle). Upon further increase in deformation, the two $\sqrt{1}$ peaks normal to the SD, (1010) and (1010), move away from the origin and lose intensity. In Figure 8c, at 140\% strain, these two peaks are very weak, but they later regain relative strength, as they move beyond the zero of the form factor (Figure $8 d)$. The peaks at higher values of $q$ are more difficult to observe. Those along the stretching direction become obscured by the first zero of the form factor as they move toward the origin, while those near the equator (normal to SD) move away from the origin and become obscured by the second form factor zero. Those peaks neither along nor normal to the SD are collapsed toward the qy axis at large $q_{y}$ (see Figure 8, parts $b$ and $c$ ). The elliptical shape of the diffraction "rings" at low deformations ( $<100 \%$ ) may still be discerned at higher deformations if one connects the peaks belonging to the same family (e.g., the $\sqrt{1}$ peaks in Figure $8 d$ ). At these large 


\section{Parallel View}

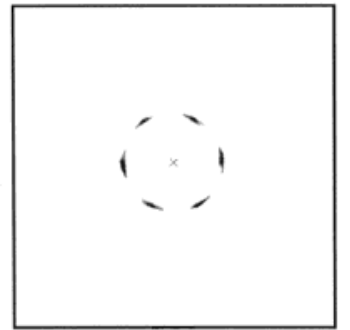

(a)

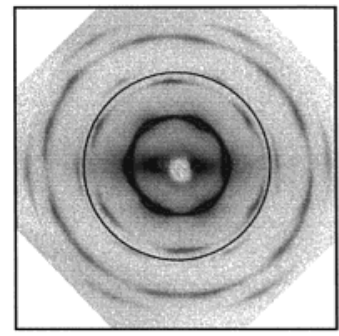

$0 \%$

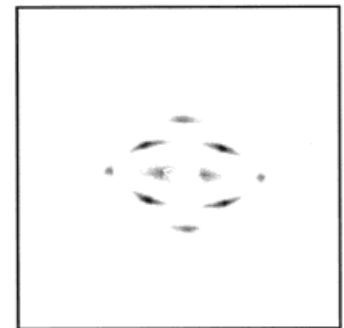

(b)

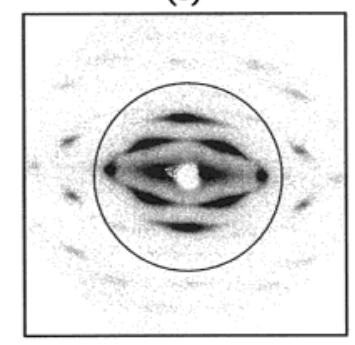

$60 \%$

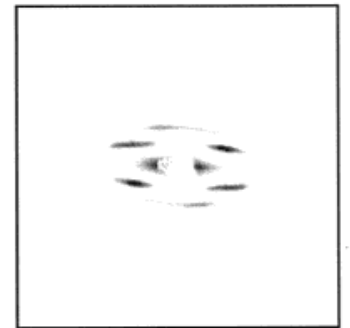

(c)

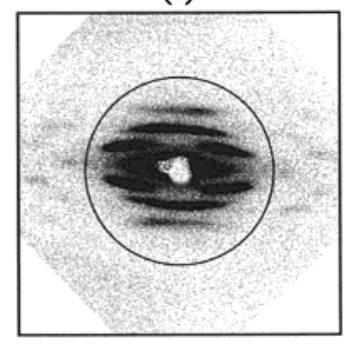

$145 \%$

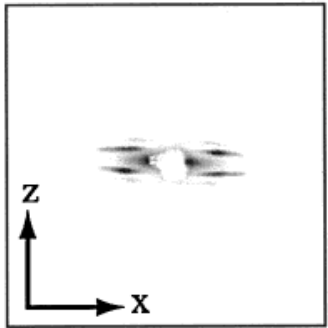

(d)

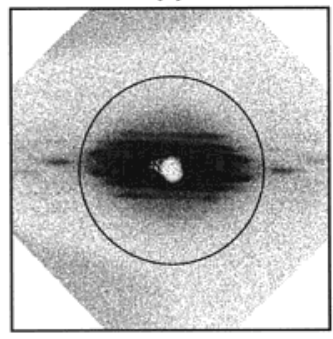

$370 \%$

Figure 8. 2-D SAXS as a function of strain for an SIS triblock cylinder morphology viewed parallel to the cylinder axis (Figure 1). The initial hexagonal pattern (a) distorts continuously with increasing deformation. The circle of radius $q=0.046 \AA^{-1}$ represents the location of the zero of the form factor. Note how the (1010) and (1010) peaks disappear into the form factor zero in part c and reemerge in part $\mathrm{d}$.

deformations, however, these ellipses have very large aspect ratios (ratio of the major/minor axes).

\section{Discussion}

5.1. Small Strain Regime. 1-D traces of the intensity along the SD for the perpendicular view data set of Figure 6 are shown in Figure 9a. The relative intensity of the $\sqrt{1}$ peak decreases while that of the $\sqrt{3}$ peak grows. The intersection of the peak position with the E wald sphere and the location of the peak relative to the zero of the cylinder form factor account for this effect. The two pairs of $\sqrt{1}$ peaks nearest the SD

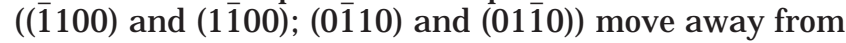
the Ewald sphere (here, the Xz plane) (Figure 8) so that the intensity observed in the perpendicular view (line $\mathrm{AA}^{\prime}$ in Figure 4$)$ decreases. The (1210) and (1210) peaks simultaneously grow in intensity as they move away from the zero of the form factor and toward the origin. The intensity of the other peaks is strongly modulated during the course of the deformation as they move toward or away from the zero of the form factor. If the relative peak intensities are taken as a guide to the position of the form factor zero, close inspection of Figure 9a indicates that the form factor zero remains fairly constant in position. This implies that the glassy PS cylinder cross section does not change during the deformation. The same conclusion was reached for PS spheres stretched to large deformations. ${ }^{12}$

The spacing between the (1210) planes (Figure 4) can be determined from the $\sqrt{3}$ peak position of Figure 9 a. A Gaussian fit was performed for each $\sqrt{3}$ peak in Figure 9a and the data plotted in Figure 10. With

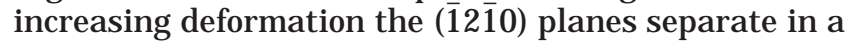
linear and nearly affine manner up to the split of the 1-D SAXS pattern into an "X" (Figure 6).

The parallel view, on the other hand, provides an opportunity to follow the distortion of the hexagonal lattice in the direction normal to the SD (y direction) via the (10ī0) peaks (Figure 4). As the (1010) peaks move away from the origin, the spacing between the (1010) planes decreases (Figure 11). The lattice becomes significantly compressed in the y direction.

With microstrain information from two orthogonal directions, the $y$ and the $z$ directions, it is possible to cross-plot the data in order to estimate Poisson's ratio in the yz plane, $v_{\mathrm{yz}}$, which is defined as the negative ratio of the lateral incremental contraction to the extension in the SD:

$$
v_{\mathrm{yz}}=\frac{-\epsilon_{\mathrm{y}}}{\epsilon_{\mathrm{z}}}
$$

where $\epsilon$ is the true strain given by

$$
\epsilon_{\text {true }}=\ln \left(\frac{d}{d_{0}}\right)
$$

For a body with orthotropic symmetry, there are six Poisson's ratios. ${ }^{35-37} \mathrm{~A}$ plot of $\epsilon_{\mathrm{y}}$ vs $\epsilon_{\mathrm{z}}$ provides $-v_{\mathrm{yz}}$ as the slope. The slope in Figure 12 yiel ds a Poisson ratio of 0.91 . As the data in the y direction are sparse, only a few points are available.

An analysis of data from Hadziioannou et al. provides confirmation of this unusual result. ${ }^{21}$ Figure 12 also plots true strain normal to the SD vs true strain in the SD measured in both real space (actual sample dimensions) and reciprocal space. The Poisson ratios $\left(v_{\mathrm{yz}}\right)$ for the real space and reciprocal space data are 0.86 and 0.91 , respectively. The available data indicates that the Poisson ratio in the $y-z$ plane for this oriented cylindrical microcomposite is closer to 1.0 than the usual value of 0.5 for isotropic rubbery materials. The significance of this result will be discussed in section 6.1. 


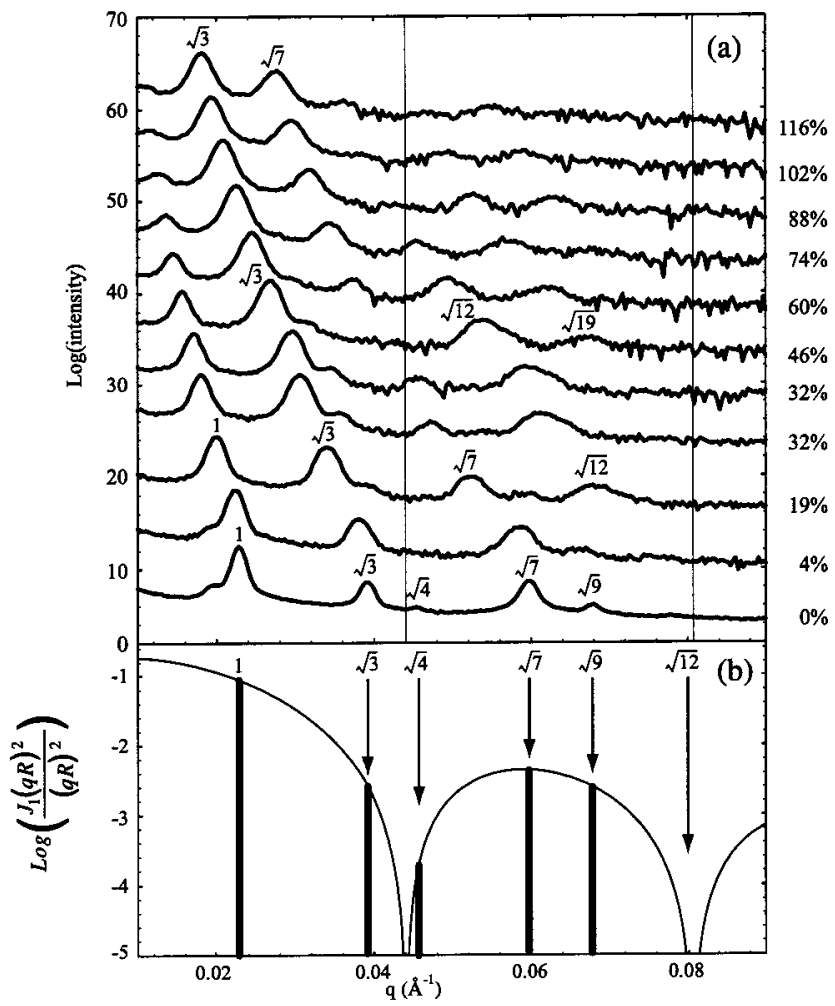

Figure 9. (a) Plot of log(intensity) vs $q_{z}$ (along SD) for select SAXS patterns of the perpendicular view data set shown in Figure 6 . Traces were obtained by integrating pie-shaped wedges (Figure $3 a$ ) or thin rectangles aligned with the SD. Both methods were performed to yield the two traces at $32 \%$ strain. The average strains during data acquisition are indicated and the plots have been offset for clarity. The first and second form factor zeros for a cylinder of radius $=83 \AA$ are indicated by the thin vertical lines. (b) Multiplication of the square of the form factor (solid thin line) with the lattice factor (solid thick lines) results in the 1-D scattering function shown above for an unoriented sample. The curve is constructed for a cylinder with a radius $R=83 \AA$ and a lattice constant of $310 \AA$ consistent with the morphology investigated. The reflection ratios label the peak positions with increasing q. Note that the $\sqrt{12}$ peak is suppressed by the second zero of the form factor.

5.2. Transition Regime. The transition between the low strain regime of the deformation and the high strain region is characterized by the splitting of the 1-D SAXS profile in the perpendicular view (Figure 6d). The peak width in the perpendicular view continually broadens until two centroids of intensity can be resolved. The splitting of the $\sqrt{3}$ peak (Figure 3 ) is presented as a series of traces along the $x$ axis across the peak maximum as a function of engineering strain in Figure 13. The exact point at which the broad streaks localize into pairs of distinct peaks upon loading is difficult to establish, but is at a strain $<130 \%$. Another estimate for the transition strain can be made by considering Figures 10a and 11. In both cases extrapolations of the d-spacing dependence within the pre and post-split deformation regimes yield transition strains of approximately $115 \%$ and $100 \%$, respectively. Note that the sample in Figure 11 had a smaller cross-sectional aspect ratio than that in Figure 10 . Comparison with the stress-strain curves (Figures 5 and 7) suggests that widespread kinking as measured by the transition strain $(100 \%-130 \%)$ occurs just after the bend in the loading curve. It seems reasonable to suspect that the

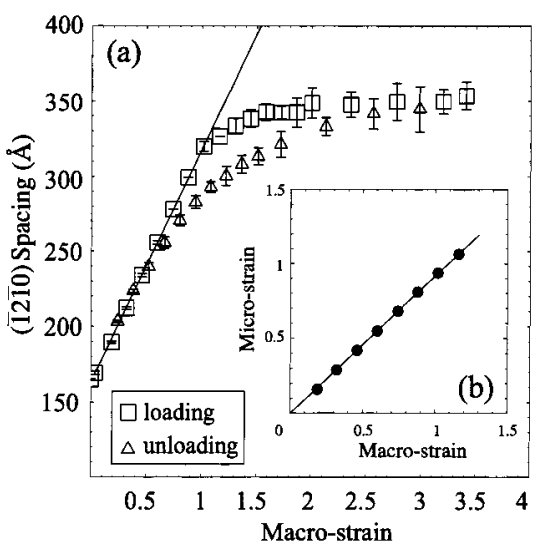

Figure 10. (a) Interplanar spacing of the ( $\overline{1} 2 \overline{1} 0)$ peak which corresponds to the $\sqrt{3}$ peak of Figure 3 a. Here, the deformation process can be divided into two stages, an initial linear regime of increasing intercylinder spacing and a later regime of fairly constant spacing. The onset of extensive kinking is estimated to be between 100 and $130 \%$ strain. (b) Engineering microstrain plotted as a function of applied macrostrain. A slope of 0.92 indicates a high degree of affineness in the deformation up to a strain of $120 \%$.

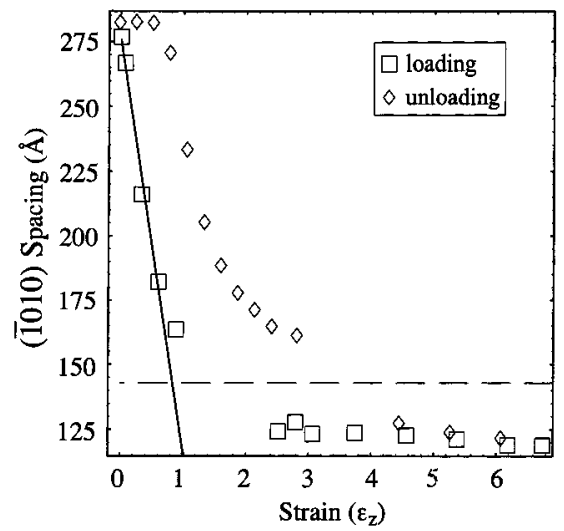

Figure 11. Interplanar spacing corresponding to the ( $\overline{1} 010)$ peak normal to the SD for the data set of Figure 8 plotted as a function of applied engineering strain. The lattice becomes compressed al ong y as observed by the linear decrease in the (1010) interplanar spacing. At higher deformations (>120\%) the spacing remains fairly constant. The boxes represent loading while the diamonds represent unloading. These data indicate a two-stage process in the lateral (y) dimension of the distorted hexagonal lattice. The horizontal dashed line shows position of zero of the cylinder form factor which causes loss of (1010) peak intensity in its vicinity (solid rings in Figure 8).

onset of localized kinking occurs earlier as indicated by the broadened of the peaks in Figure 6c.

5.3. Large Strain Regime. After the $\sqrt{3}$ peaks split into an X-pattern, the arms of the "X" rotate away from each other with increasing deformation. Figure 14a tracks the angle between the arms of the " $X$ " as a function of deformation. The $X$ is first resolved at approximately $130 \%$ strain at an angle of $50^{\circ}$ and continues to increase as far as the sample is stretched (Figure 14b). At the highest deformations, the rate of increase levels off and becomes asymptotic. U pon unloading, the $X$ is observed down to an angle of $10^{\circ}$ and a strain of $25 \%$. The hysteresis of the X-arm motion during a loading/unl oading cycle begins at strains less than $200 \%$. The unloading portion of the X-angle curve is linear and extends almost back to the origin in contrast to the loading portion, which begins only after a strain of approximately $130 \%$. 


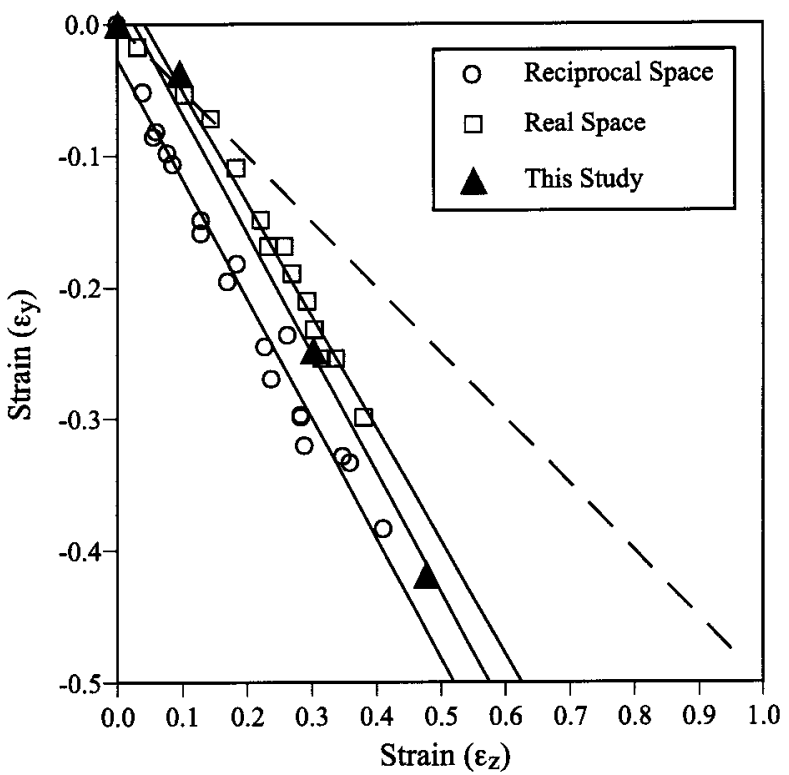

Figure 12. Plot of $\epsilon_{y}$ (microstrain along y) vs $\epsilon_{z}$ (microstrain along $z$ ). The negative slope of the filled symbols yields a Poisson ratio of 0.91 . The open symbols represent a perpendicular stretch experiment on a 7.5-35-7.5 molecular weight SIS triblock of $\mathrm{Hadzi}$ ioannou et al. ${ }^{21}$ Both direct space measurements of the sample dimensions (diamonds) and reciprocal space measurements of the lattice (circles) have nearly the same slope. The real space fit yields a Poisson ratio of 0.86 , whereas the reciprocal space data fit yields a Poisson ratio of 0.91 . The dashed lines indicate Poisson ratios of 0.5 and 1 , respectively.

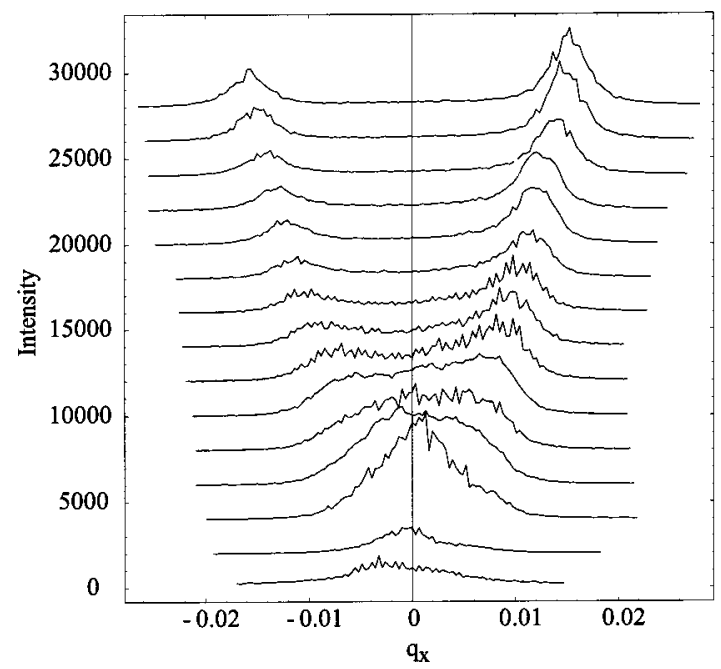

Figure 13. Intensity vs $\mathrm{q}_{\mathrm{x}}$ for the $\sqrt{3}$ peak obtained by placing a rectangle around the peak and integrating along $z$ (SD). The peak split is readily resolved at a strain of $130 \%$, but begins to form earlier. The strong initial increase in the peak intensity is due to the modulation of the moving peak by the invariant cylinder form factor.

The 2-D SAXS patterns describing the progression of deformation in the perpendicular incidence geometry can be quantified by considering the peak positions as a function of the deformation. Though the position of the centroid of a peak may not always be the same as the position of the peak maximum, in the following it is the position of the peak maximum that is reported. Figure 14c shows the trace of the position of the $\sqrt{3}$ peak for the deformation run described previously. The direction of the deformation is indicated by arrows. The $\sqrt{3}$ peak moves toward the origin during the loading
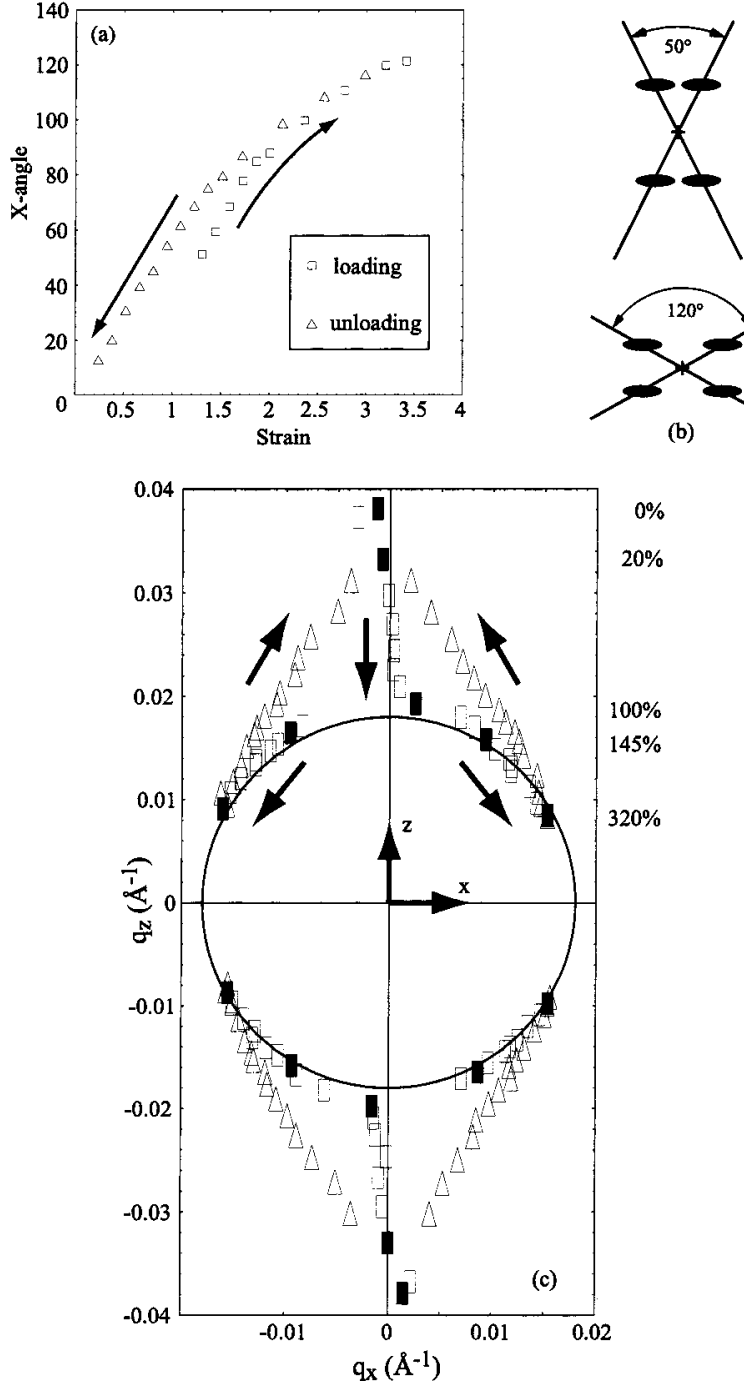

Figure 14. (a) Plot of the angle between the $\sqrt{3}$ peaks in an " $X$ " pattern as a function of deformation. (b) Schematic diagrams of the extremes of the positions of the split $\sqrt{3}$ peak on loading. (c) Trace of $\sqrt{3}$ peak position during a loading/ unloading cycle at perpendicular incidence. The arrows indicate the direction of peak movement during the deformation. A circle of radius $0.018 \AA^{-1}$ has been placed about the origin to indicate how after $X$ pattern formation the $\sqrt{3}$ peak follows the arc of a circle. The filled points represent data from the patterns in Figure 8.

cycle and as shown in Figure 6, then splits apart to form an X-pattern. Upon unloading, the peaks follow a different, more direct path back towards the initial point of the deformation. The figure shows a slight asymmetry about the stretching direction which is also reflected in the 2-D SAXS patterns (Figure 6). It has been found to be difficult to align the morphology with the stretching direction to give a perfectly symmetric X-pattern during a perpendicular stretch. Upon kinking, the amount of hysteresis in the path of deformation increases. The further along the circle in Figure 14c that the $\sqrt{3}$ peaks travels on loading and the more time spent at load, the more irreversible the deformation as described by the recovery path (triangles in Figure 14c).

\section{Deformation Mechanism}

6.1. Small Strain. In light of the available data, a mechanism is proposed to explain the deformation 
Table 1. Studies of Affiness in Perpendicular Deformation Experiments on Oriented Cylinders

\begin{tabular}{|c|c|c|c|c|}
\hline ref & deformation affine? & $\begin{array}{l}\text { maximum strain of } \\
\text { affine behavior }\end{array}$ & $\begin{array}{l}\text { maximum } \\
\text { strain } \\
\text { investigated }\end{array}$ & material \\
\hline $\begin{array}{l}\text { Odell and Keller, } 1977^{18} \\
\text { Hadziioannou et al., } 1977^{21,47} \\
\text { Pakula et al., } 1985^{24} \\
\text { Fairclough et al., } 2000^{25} \\
\text { this study }\end{array}$ & $\begin{array}{l}\text { yes } \\
\text { yes } \\
\text { "uniform extension until yield point" } \\
\text { no } \\
\text { nearly affine }\left(v_{\mathrm{yz}}=0.9\right)\end{array}$ & $\begin{array}{l}20 \% \\
50 \% \\
\text { yield point (between } 20 \text { and } 70 \% \text { ) } \\
6.7 \% \\
100-130 \%\end{array}$ & $\begin{array}{l}50 \% \\
50 \% \\
500 \% \\
6.7 \% \\
700 \%\end{array}$ & $\begin{array}{l}\text { SBS } \\
\text { SIS } \\
\text { SBS } \\
\text { S-EB-Sa } \\
\text { SIS }\end{array}$ \\
\hline
\end{tabular}

process during a perpendicular stretching experiment. As the sample begins to deform, the cylinders move apart al ong $z$ in a nearly affine manner. The SAXS data taken with the X-ray beam at perpendicular incidence show an increase in spacing between cylinders in the SD, which is nearly equal to the macroscopic applied strain (Figure 10b). The rubber matrix, being intimately connected to the PS cylinder via covalent bonds, follows the movement of the cylinders. The cylinders therefore, act as markers for the deformation of the matrix up until the onset of kinking.

As the rubber stretches in the SD, it contracts in the lateral direction in an attempt to preserve vol ume. This lateral contraction can be monitored by the movement of the Bragg peaks arising from the (1010) and (1010) planes al ong the equator (Figure 4). Data from Figure 12 show that the lateral contraction in the $y-z$ plane is larger than that expected for an ideal rubber. This initially somewhat surprising result can be readily understood if the anisotropy of the structure is kept in mind. While the matrix is being stretched in the SD, it attempts to contract in the plane normal to the SD in order to preserve volume. Since the glassy cylinders are all oriented in the $x$ direction, the matrix is highly constrained in this direction. The direction of least resistance to contraction is therefore the y direction, which then experiences a preferential contraction much greater than that of an unconstrained isotropic rubber.

For an ideal fiber-reinforced composite of perfectly oriented incompressible fibers (oriented along the $x$ direction) perfectly adhering to a rubbery matrix, one can anticipate that for every unit of perpendicular extension (along the $z$ direction), one unit of lateral contraction (along the $y$ direction) would result. The Poisson ratio in the $y-z$ plane would be equal to 1 , while the Poisson ratio in the $x-z$ plane would equal 0 . Hence, the rubbery matrix would continue to undergo a constant vol ume deformation. Because of the fibers aligned in the $x$ direction, the matrix deformation is constrained to the $y-z$ plane. Here, the data from the present study as well as that available from the literature $\mathrm{e}^{21}$ indicates that $v_{\mathrm{yz}}$ has a value of approximately 0.9 (Figure 12). Finally, it should be noted that this experimental result was anticipated by some of those involved in the earliest studies of oriented cylinders morphology. ${ }^{17}$

Several possible reasons exist for why the experimentally measured Poisson ratio does not equal that of the ideal case. Perhaps the most significant of these is the misalignment of the cylinder axis with respect to the compression direction. If the cylinder normals are not perfectly aligned along the SD, the effectiveness of the cylinders in resisting the compressive force of the matrix is drastically reduced. Sample preparation and alignment may also exacerbate this problem; e.g., the tensile specimen may be imperfectly mounted with respect to the SD. Furthermore, the average cylinder normal within the tensile specimen may not be aligned with the sample's long axis due to misalignment of the die used to stamp out the tensile specimen from the rollcast film. Indeed, a perfectly symmetric X-pattern during a perpendicular deformation experiment is rare (Figure 6). The consequence of cylinder misalignment is that any change in the dimension of the sample in the $x$ direction reduces the need for the matrix to contract exclusively in the y direction, thereby reducing the Poisson's ration in the $y-z$ plane.

The constraint in deformation al ong the $x$ direction arises not only from the anisotropic morphology but also potential ly from the sample geometry itself. Indeed, the difference in sample cross section for the two tensile specimens under consideration partially accounts for the difference in strain at the kinking transition. This effect can be understood in terms of the limiting behavior of elastically deformable materials. A sample of infinite width and infinitesimal thickness would be in a state of plane strain under deformation as the width dimension would not be able to change. ${ }^{38}$ As the cross-sectional aspect ratio approaches 1 , the deformation behavior is better characterized as uniaxial. Since the morphological constraint operates in the width direction, the plane strain condition is accentuated.

A slope of 0.9 in the relationship between lattice deformation and sample deformation in Figure 10b strongly suggests a nearly affine deformation. The affineness of the deformation is connected to Poisson's ratio. With this particular morphology and this particular deformation geometry, perfectly affine deformation would require a Poisson ratio $v_{\mathrm{yz}}$ of 1 . This affineness is found to persist to a deformation of about $120 \%$ (Figure 10), at which point the morphology undergoes widespread kinking. Other authors have investigated the extent of affineness in similar samples with various results as can be seen in Table 1 . The strain at which the deformation is no longer observed to be affine is that at which kinking becomes widespread. Onset of widespread kinking, in turn, depends critically on the extent and quality of global orientation.

6.2. Transition Strain. The transition between the initial affine deformation at strains $<100 \%$ and the later large strain deformation at strains $>130 \%$ represents the regime in which the oriented cylinders kink into a chevron pattern. Although the present experimental data is not sensitive to precisely what happens during the transition, a tentative mechanism consistent with the available data at low and high strains is nonetheless proposed. As the axial stress on the PS cylinders increases, locally misaligned regions (due to defects) are no longer able to support the compression. A moment between the applied compressive force and the component of cylinder misalignment develops and those moments along the $y$ axis are promoted at the expense of all others as their development relieves stress in the SD. The result is that the misaligned cylinders turn predominantly clockwise or counterclockwise in the 


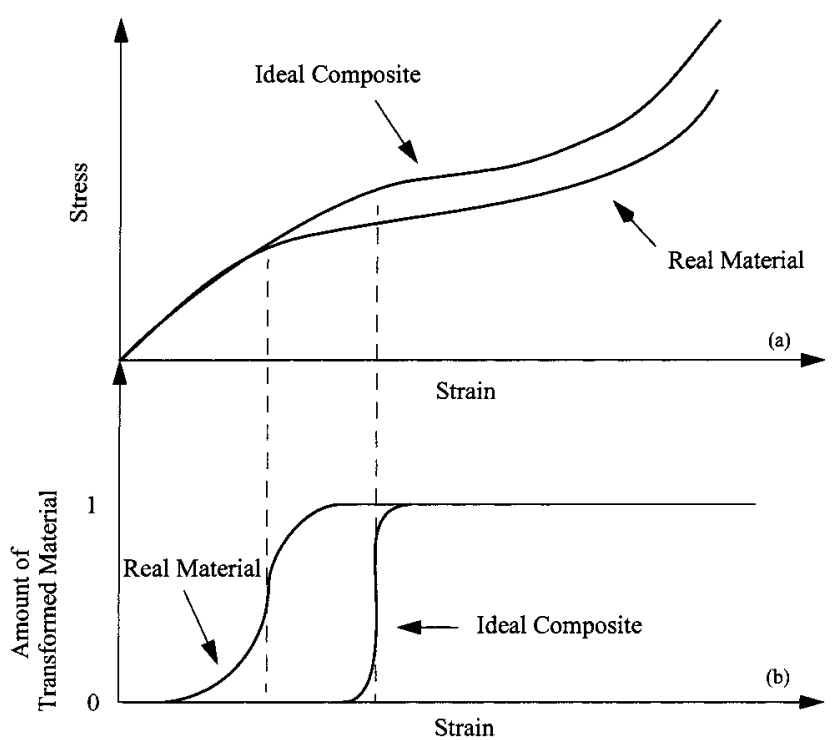

Figure 15. (a) Schematic stress-strain plot for a perfect composite vs a real material in perpendicular deformation. The stress-strain curve up until the kinking transition is equivalent to a rubber in a plane strain condition. The release of this condition occurs at lower strains in a real material as compared to an ideal system due to defects. (b) Schematic pl ot of the fraction of material that has undergone kinking. The ideal composite transforms catastrophically, while the transformation of the real material is more continuous.

$x-z$ plane. Resisting this autocatalytic increase in bending of the cylinders is the shear resistance of the rubber and the plastic work associated with bending the PS rods. As the cylinders turn, the rubber matrix between cylinders undergoes shear. This shear resistance helps to prevent the misaligned cylinders from kinking immediately upon application of the compressive stress. Since the stress-strain curve does not exhibit a sharp yield point in shear, a specific shear strength for the rubber matrix does not exist. The shear resistance of the rubber matrix increases nonlinearly.

Regions of varying degrees of misalignment are scattered throughout the volume of the sample. The kinking process therefore cannot occur everywhere simultaneously, but develops in a more continuous manner. Those regions of greater misalignment will nucleate kinks and these grow first, whereas well-aligned regions resist kinking. The sharpness of the kinking transition is expected to depend strongly on the number, nature, and degree of misorientation of the aligned cylinders.

In contrast, for a perfectly aligned microdomain composite, upon reaching the critical stress, kinks will form catastrophically. From a continuum standpoint, the stress-strain curve of such an ideal sample would follow the elastic-perfectly plastic model as has been suggested. ${ }^{26}$ The cylinders are stretched apart affinely until the critical strain at which the cylinders cooperatively kink. However, a continuum treatment ignores the impact of the chain stretching of the matrix rubber which controls the shape of the stress-strain curve in this regime. Here, the stress-strain curve is best modeled as a constrained rubber in plane strain. The material would reversibly deform by following the stress-strain curve of a rubber in plane strain conditions up until the critical kinking stress. The stressstrain response of an ideal composite would carry the constrained rubber to higher strains (and stresses) before kinking (Figure 15) as the ideal composite would be free of kink-inducing defects. After catastrophic kinking the deformation would proceed irreversibly as the kinked PS rods continued bending.

The kinking transition is most easily observed via SAXS with the incident beam perpendicular to the cylinder axis (Figure 6, also see Figure 13). With the incident beam parallel to the cylinder axis, close inspection of the movement of the (1010) peak (Figure 11) as a function of deformation also reveals a discontinuity at a strain value nearly that found for the perpendicular view (Figure 10a). As the hexagonal lattice is compressed laterally, the cylinders move closer together. The constraint along $x$ due to the oriented cylinders is removed upon widespread kinking. At this point the rate of contraction along y almost drops to zero as revealed in the fairly constant (1010) interplanar spacing of Figure 11 at strain $>120 \%$. Thus, the kinking transition makes itself apparent in the movement of the cylinders in the y direction which can only be followed with SAXS at parallel incidence.

A recent 2-D theoretical study predicts chevron formation to occur via buckling. ${ }^{26}$ The glassy component profile at the instability ( $3 \%$ strain) is modeled as a sinusoid which then transforms to a chevron pattern at higher (10-20\%) strains. The main difference to our system within the small strain regime is the extent of the affine deformation. Whereas the 2-D model of Read et al. predicts affine deformation up to a strain of $3 \%$, we measure affine deformation extending at least to $100 \%$, due in large part of the ability of the structure to contract in the third (y axis) direction.

6.3. Large Strain. After formation of the well-defined X-pattern at approximately $130 \%$ strain (Figure 13), further motion of the $\sqrt{3}$ peaks occurs about the origin along the trace of a circle (Figure 14c). This motion indicates a rotation of the scattering objects at constant q. TEM data ${ }^{27}$ demonstrate, however, that the kinked cylinders turn toward the SD via a shearing process. These two different statements about the movement of the cylinders during deformation beyond kinking are combined into a deformation mechanism involving simultaneous shear and dilation al ong the SD.

Figure 16 distinguishes the two cases of pure rotation and pure shear in real and reciprocal space. Pure rotation requires that qrot remains constant, while for pure shear, $\mathrm{q}_{\text {shear }}$ increases with $\theta$. The model, which accounts for both SAXS and TEM data, combines a shearing mechanism with a concurrent increase in $d$ spacing so that the intercylinder spacing remains constant. Figure 17 illustrates this shearing-plus-dilation model.

\section{Conclusions}

A poly(styrene-isoprene-styrene) triblock copolymer with a cylinder morphology was oriented via roll-casting. The extent of orientation was characterized by SAXS with the incident beam parallel and perpendicular to the cylinder axis. The azimuthal width of the Bragg peaks at perpendicular incidence indicated that most of the rods were oriented parallel within $15^{\circ}$ of one another (misorientation about y). At parallel incidence the rotational misorientation of the cylinders was measured to be approximately $11-13^{\circ}$ (misorientation about $\mathrm{x}$ ). The system, though not a single crystal, has a much higher degree of orientation than that of a uniaxial fiber. 


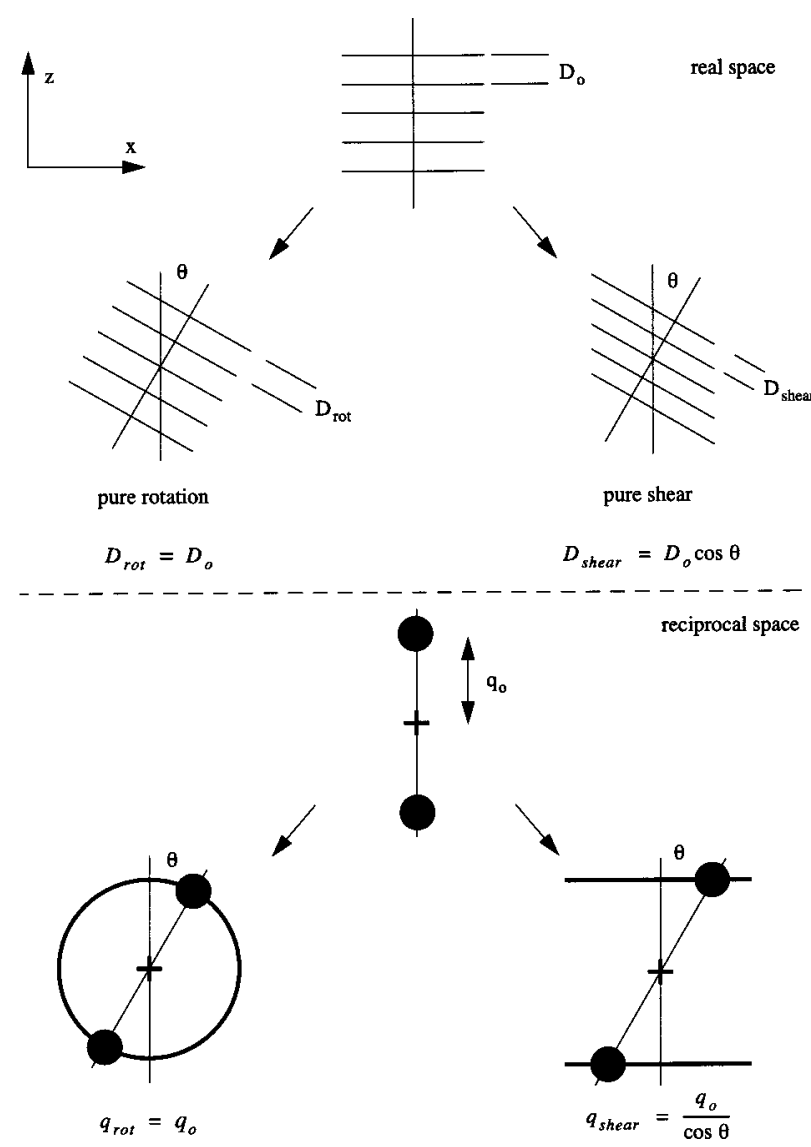

Figure 16. Schematic diagram distinguishing pure rotation (left) and pure shear (right) in real space (top) and reciprocal space (bottom) for a stack of rods of initial spacing $D_{0}$.

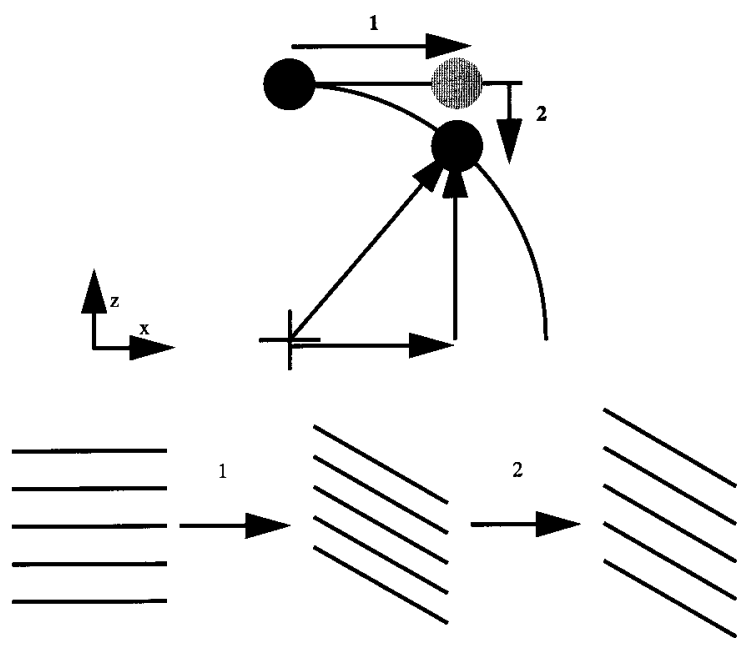

Figure 17. Schematic of the post-kink deformation process at perpendicular incidence. SAXS shows that after kinking, the cylinders turn into the SD without a change in the intercylinder distance (Figure 14c). The cylinders shear into the SD (step 1) and simultaneously dilate (step 2). This shearing-plus-dilation model accounts for the motion of the $\sqrt{3}$ peak along a circle as well as the chevron pattern in the TEM images. ${ }^{27}$

On the basis of the 2-D SAXS patterns obtained with the incident beam along $y$, two regimes of deformation can be distinguished. At strains up to approximately $100 \%$, the pattern remains essentially one-dimensional. The peaks move in toward the origin and change in intensity depending on their location with respect to the zero of the form factor and E wald sphere. The initial intercylinder spacing of $310 \AA$ increases to approximately $680 \AA$. At $100-130 \%$ deformation the peaks broaden and split to form an X-pattern. Thereafter the angle between the arms of the $X$ increases asymptotically, and the peaks follow the a circular path about the origin indicating a constant intercyinder spacing at about $680 \AA$. The loading/unloading path of deformation is dissimilar, the kinked cylinders straighten proportionally to the extent of unloading (Figure 14), consistent with the hysteresis.

2-D SAXS patterns taken with the incident beam along the cylinder axis (parallel view) show a continuous distortion with increasing deformation. The $6 \mathrm{~mm}$ symmetry associated with the hexagonal packing of the lattice is immediately reduced to $2 \mathrm{~mm}$ symmetry. The motion of the (1010) reflections oriented along the equator show a discontinuity at a strain nearly equal to the $\mathrm{X}$ pattern transition strain found in the perpendicular view.

In combination with TEM data reported elsewhere, ${ }^{27}$ the SAXS data allow a description of the deformation process. The low strain regime is associated with a nearly affine separation of the cylinders al ong $z$ (the SD) together with a lateral contraction along y (the neutral direction). The extension along $z$ is almost completely balanced by the lateral contraction in the y direction. The Poisson's ratio in the $y-z$ plane is measured to be approximately 0.9 . This result is interpreted in terms of a constrained rubber undergoing constant volume deformation. The deviation of the measured Poisson's ratio from that of a perfectly oriented specimen $\left(v_{\mathrm{yz}}=\right.$ 1 ) is primarily attributed to morphological defects and sample misorientation.

The onset of a kinking instability in which the cylinders kink into a chevron pattern is ascribed to regions of misoriented cylinders which can no longer support the compressive stress along $x$. The finite shear resistance of the rubber matrix as well as the resistance of the PS rods to bending prevents these regions from undergoing this kinking stability immediately. The $\mathrm{X}$-ray peak broadening at the kinking transition is interpreted as a distribution of cylinder segments which have locally kinked to different extents.

At higher strains the kinked cylinders undergo simultaneous shear and dilation as they turn toward the stretching direction. This combination of deformation modes is most consistent with both the SAXS data which suggests cylinder rotation and the TEM data (reported elsewhere) ${ }^{27}$ which suggests shear. The cylinders remain well ordered and highly cooperative at even the highest strains as evidenced by SAXS patterns showing up to seven reflections.

Having identified the kinking transition as a critical step in the perpendicular deformation of highly oriented cylinders, future work could focus on determining the sensitivity of the transition strain to various material parameters such as molecular weight, moduli of the components, defects, and orientation of the structure. Modeling might attempt to include the influence of the rubber matrix on the kinking transition in terms of a entropic network in plane strain. Further elucidation of the deformation mechanism is expected by examining the role of the bridging blocks. ${ }^{39}$ The feasibility for the determination of the molecular orientation of both the PS and rubbery midblocks has been shown for unoriented samples using infrared dichroism. ${ }^{40,41}$ Wide-angle X-ray scattering (WAXS) indicates that the rubbery 
chains become highly oriented in the stretching direction at the highest deformations. ${ }^{41}$

The understanding gained by investigating the deformation of highly oriented styrene-diene triblock copolymer systems is expected to aid in the correct interpretation of the influence of chemistry on the mechanical properties of block copolymers. Indeed, improved mechanical properties motivate the preparation of triblocks of different chemical identity. ${ }^{42-46}$ However, without the ability to separate the influence of morphology from chemistry on the mechanical properties, the correlation of morphological and chemical structure with mechanical properties will remain simply a correlation.

Acknowledgment. This work was supported by grants from NSF: DMR 98-01759 and MRSEC MIT facilities. C.C.H. thanks Prof. A. Argon and Prof. D. C. Martin for hel pful discussions. Dr. B. Walters of Dexco is acknowledged for providing the material.

\section{References and Notes}

(1) Creemers, H. M. J . C. Kunststoffe 1996, 86, 1845.

(2) Rader, C. P. Modern Plastics: Encyclopedia 1997; McGrawHill Co.: New York, 1996; Vol. 73; p B51.

(3) Lauhus, W. P.; Haberstroh, E.; Ehrig, F. Kunststoffe 1997, 87, 706.

(4) Mieras, H. J . M. A.; Wilson, E. A. J . Inst. Rubber Ind. 1973, 7, 72.

(5) Folkes, M. J .; Reip, P. W. Polymer 1986, 27, 377.

(6) Leist, H.; Geiger, K.; Wiesner, U. Macromolecules 1999, 32, 1315.

(7) Bates, F. S.; Schulz, M. F.; Khandpur, A. K.; Förster, S.; Rosedale, J. H.; Almdal, K.; Mortensen, K. Faraday Discuss. 1994, 98, 7.

(8) Bates, F. S.; Fredrickson, G. H. Annu. Rev. Phys. Chem. 1990, $41,525$.

(9) Bates, F. S.; Fredrickson, G. H. Phys. Today 1999, Feb., 32.

(10) Winey, K. I.; Gobran, D. A.; Xu, Z.; Fetters, L. J .; Thomas, E. L. Macromolecules 1994, 27, 2392.

(11) de la Cruz, M. O.; Sanchez, I. Macromolecules 1986, 19, 2501.

(12) Prasman, E.; Thomas, E. L. J Polym. Sci., Part B 1998, 36, 1625.

(13) Dair, B. J .; Avgeropoulos, A.; Hadjichristidis, N.; Thomas, E. L. J . Mater. Sci. 2000, 35, 5207. Dair, B. J .; Avgeropoulos, A.; Hadjichristidis, N.; Capel, M.; Thomas, E. L. Polymer 2000, 41, 6231.

(14) Cohen, Y.; Albalak, R. J .; Dair, B.J .; Thomas, E. L.; M. Capel Macromol ecules 2000,33, 6502.

(15) Keller, A.; Odell, J . A. Processing, Structure and Properties of Block Copolymers; Elsevier Applied Science: New York, 1985; Chapter 2.

(16) Folkes, M. J .; Keller, A.; Scalisi, F. P. Colloid Polym. Sci. 1973, 251, 1.

(17) Arridge, R. G. C.; Folkes, M. J . J . Phys. D: Appl. Phys. 1972, $5,344$.
(18) Odell, J . A.; Keller, A. Polym. Eng. Sci. 1977, 17, 544.

(19) Hadziioannou, G.; Mathis, A.; Skoulios, A. Colloid Polym. Sci. 1979, 257, 136.

(20) Hadziioannou, G.; Mathis, A.; Skoulios, A. Colloid Polym. Sci. 1979, 257, 15.

(21) Hadziioannou, G.; Mathis, A.; Skoulios, A. Colloid Polym. Sci. 1979, 257, 337.

(22) Tarasov, S. G.; Tsvankin, D. Y.; Godovsky, Y. K. Polym. Sci. USSR 1978, 20, 1728

(23) Kitaigorodsky, A. I. Mixed Crystals; Springer: Berlin, 1984; p 332.

(24) Pakula, T.; Saijo, K.; Kawai, H.; Hashimoto, T. Macromolecules 1985, 18, 1294.

(25) Fairclough, J. P. A.; Salou, C. L. O.; Ryan, A. J .; Hamley, I. W.; Daniel, C.; Helsby, W. I.; Hall, C.; Lewis, R. A.; Gleeson, A. J .; Diakun, G. P.; Mant, G. R. Polymer 2000, 41, 2577.

(26) Read, D. J .; Duckett, R. A.; Sweeney, J .; McLeish, T. C. B. J . Phys. D: Appl. Phys. 1999, 32, 2087.

(27) Honeker, C. C.; Thomas, E. L. Macromol ecules 2000, 33, 9407.

(28) Albalak, R. J .; Thomas, E. L. J Polym. Sci. B: Polym. Phys. 1994, 32, 341.

(29) Annighoefer, F.; Gronski, W. Makromol. Chem. Rapid Commun. 1983, 4, 123.

(30) Albalak, R. J . Polymer Devolatilization; Marcel Dekker: New York, 1996.

(31) Albalak, R. J . Polymer 1994, 35, 4115

(32) Craven, B. M.; DeTitta, G. T. J . Chem. Soc., Perkin Trans. 2 1976, 814.

(33) Buerger, M.J . Elementary Crystallography: An Introduction to the Fundamental Geometrical Features of Crystals; J ohn Wiley and Sons Inc: New York, 1956; p 528.

(34) Honeker, C. Ph.D. Thesis; Massachusetts Institute of Technology 1997

(35) Ward, I. M. Mechanical Properties of Solid Polymers; J ohn Wiley \& Sons: New York, 1975; p 475.

(36) Kim, K. Y. Phys. Rev. B 1996, 54, 6245.

(37) J ones, R. M. Mechanics of Composite Materials; McGrawHill: New York, 1975; p 300.

(38) Chou, P. C.; Pagano, N. J . Elasticity: Tensor, Dyadic, and Engineering Approaches; Van N ostrand: Princeton, NJ , 1967; p 290.

(39) Karatasos, K.; Anastasiadis, S. H.; Pakula, T.; Watanabe, H. Macromolecules 2000, 33, 523.

(40) Zhao, Y. Macromolecules 1992, 25, 4705.

(41) Sakamoto, J .; Sakurai, S.; Doi, K.; N omura, S. Polymer 1993, 34, 4837.

(42) Neumann, C.; Loveday, D. R.; Abetz, V.; Stadler, R. Macromolecules 1998, 31, 2493.

(43) Arnold, M.; Hofmann, S.; Weidisch, R.; Michler, G. H.; Neubauer, A.; Poser, S. Macromol. Chem. Phys. 1998, 199, 31.

(44) Balsamo, V.; von Gyldenfeldt, F.; Stadler, R. Macromolecules 1999, 32, 1226.

(45) Brinkmann-Rengel, S.; Abetz, V.; Stadler, R.; Thomas, E. L. Kautsch. Gummi Kunstst. 1999, 52, 806.

(46) Weidisch, R.; Stamm, M.; Schubert, D. W.; Arnold, M.; Budde, H.; Horing, S. Macromolecules 1999, 32, 3405.

(47) Hadziioannou, G. Ph.D. Thesis; Louis Pasteur University, Strassbourg, France 1977.

MA000593Y 